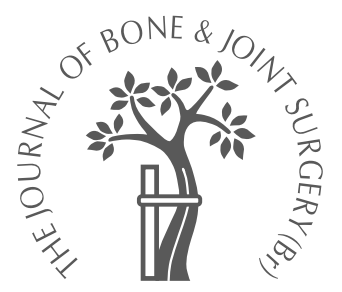

M. Kamegaya,

T. Saisu,

N. Ochiai,

J. Hisamitsu,

H. Moriya

From Chiba

Children's Hospital, Chiba City, Japan
M. Kamegaya, MD, Chief Staff Surgeon

T. Saisu, MD, Staff Surgeon

N. Ochiai, MD, Resident Division of Orthopaedic Surgery, Chiba Children's Hospital, 579-1 Heta-chou, Chiba City 266-0007, Chiba, Japan.

H. Moriya, MD, Professor J. Hisamitsu, MD, Resident Department of Orthopaedic Surgery, Chiba University School of Medicine, 1-8-1 Inohana, Chuo-ku, Chiba City 260-0856, Chiba, Japan.

Correspondence should be sent to Dr M. Kamegaya.

(C) 2004 British Editorial Society of Bone and Joint Surgery doi:10.1302/0301-620X.86B8 $14458 \$ 2.00$

$J$ Bone Joint Surg $[\mathrm{Br}]$ 2004;86-B:1176-81.

Received 17 April 2003;

Accepted after revision

28 October 2003

\title{
A paired study of Perthes' disease comparing conservative and surgical treatment
}

We performed a paired study of mature patients with Perthes' disease to compare the radiological results after treatment between conservatively- and surgically-treated groups. One patient was selected from each group to create the pairs for this study. Each pair was strictly matched for gender, body mass index, age at onset, stage at the first visit, necrotic area and radiological at-risk signs and each was assessed by comparing the values of six radiological measurements.

Eighteen pairs (36 hips) fitted the criteria. The radiological measurements which showed a statistically better result in the surgical groups were Mose's method, the acetabular-head index and leg-length discrepancy. There were no statistical differences in the slope of the acetabular roof and the articulotrochanteric distance. Four hips in the conservative group were in Stulberg class II, five in class III and nine in class IV. In the surgical group, 13 were in Stulberg class II, four in class III and one in class IV.

We conclude that surgical treatment improved the sphericity of the femoral head and provided greater acetabular cover, but did not reform the acetabular roof.

It was noteworthy that a greater leg-length discrepancy and a smaller articulotrochanteric distance were not seen in the surgical group. Our study suggests that surgical treatment is preferable in patients with severe Perthes' disease.

There is considerable controversy regarding the use of conservative or operative treatment in Perthes' disease. One of the reasons is that the patients selected for each treatment group are dissimilar particularly with regard to the severity of the disease. Comparative studies in the past have been carried out using simplistic and varying guidelines. ${ }^{1-6}$ A satisfactory comparison between conservative and surgical treatment should involve patients who are matched for severity of the disease, gender, physique, age at onset, stage at the first visit, necrotic area and radiological signs. Such an attempt has not been previously undertaken.

Our aim was to compare the radiological results at maturity in matched pairs of patients with Perthes' disease, treated either by a uniform conservative or an operative method.

\section{Patients and Methods}

We selected only unilateral patients for our study. A total of 163 patients were reviewed of whom 136 were male and 27 female. The right side was affected in 68 hips and the left in 95 . All were mature at the last follow-up. The conservative group consisted of 135 patients who had been treated before 1985 by a Tachdjiantype orthosis, which is a trilateral socket hip abduction orthosis. The surgical group consisted of 28 hips which had been treated by femoral varus osteotomy between 1990 and 1995 , in accordance with our protocol. This protocol had been created by one of the authors (MK) in $1987^{7}$ based on a long-term follow-up of the conservative group.

The protocol had three categories based on the following factors: age at onset, the Catterall classification ${ }^{8}$ and signs of severe subluxation and/or a metaphyseal cyst. Severe subluxation and a metaphyseal cyst were indicated by a difference in the teardrop distance of more than $3 \mathrm{~mm}$ between the affected and unaffected sides, and a diffuse cyst on the anteroposterior view. The categories were possibly poor, probably poor and definitely poor. Surgical intervention (varus femoral osteotomy) was undertaken in patients classified as possibly poor with persistent limitation of movement of the hip, particularly if there was less than $30^{\circ}$ of abduction, or for all patients in the probably and definitely poor groups. The desired post-operative femoral neck-shaft angle was approximately $100^{\circ}$. 
Table I. Details of the 18 pairs of patients

\begin{tabular}{|c|c|c|c|c|c|c|c|c|}
\hline \multirow[b]{2}{*}{ Number of pair } & \multicolumn{2}{|c|}{ Body mass index* } & \multicolumn{2}{|c|}{ Age at onset (yrs + mths) } & \multirow[b]{2}{*}{ Stage } & \multirow{2}{*}{$\begin{array}{l}\text { Catterall group } \\
\text { (III or IV) }\end{array}$} & \multicolumn{2}{|c|}{ Head-at-risk sign } \\
\hline & Cons & Surg & Cons & Surg & & & Subluxation & Metaphyseal cyst \\
\hline 1 & 15.9 & 17.7 & $9+1$ & $9+6$ & Necrotic & III & + & + \\
\hline 2 & 15.1 & 15.4 & $8+4$ & $8+5$ & Initial & III & + & + \\
\hline 3 & 16.2 & 16.8 & $10+0$ & $10+6$ & Necrotic & III & + & + \\
\hline 4 & 16.5 & 15.9 & $7+0$ & $7+2$ & Necrotic & III & + & + \\
\hline 5 & 15.3 & 15.2 & $6+9$ & $7+6$ & Necrotic & III & + & + \\
\hline 6 & 15.5 & 16.9 & $8+4$ & $8+3$ & Initial & III & + & + \\
\hline 7 & 14.8 & 15.1 & $6+3$ & $6+6$ & Initial & III & + & + \\
\hline 8 & 16.2 & 15.8 & $8+1$ & $8+5$ & Necrotic & III & + & + \\
\hline 9 & 17.3 & 16.2 & $7+1$ & $6+7$ & Necrotic & IV & + & + \\
\hline 10 & 17.3 & 16.4 & $10+9$ & $10+1$ & Regenerative & III & + & + \\
\hline 11 & 18.2 & 17 & $9+8$ & $9+0$ & Initial & III & + & + \\
\hline 12 & 17.6 & 15.8 & $8+1$ & $9+0$ & Necrotic & III & + & $\mathrm{n} / \mathrm{a}$ \\
\hline 13 & 16.5 & 15.6 & $8+6$ & $8+7$ & Necrotic & III & + & $\mathrm{n} / \mathrm{a}$ \\
\hline 14 & 16.7 & 18.1 & $8+1$ & $8+1$ & Necrotic & III & + & + \\
\hline 15 & 17.9 & 16.5 & $8+5$ & $8+8$ & Necrotic & III & + & + \\
\hline 16 & 18.5 & 17.9 & $10+1$ & $10+4$ & Necrotic & III & + & $\mathrm{n} / \mathrm{a}$ \\
\hline 17 & 17.8 & 17.1 & $11+0$ & $10+6$ & Regenerative & III & + & $\mathrm{n} / \mathrm{a}$ \\
\hline 18 & 17.1 & 16.8 & $11+3$ & $11+0$ & Initial & III & + & + \\
\hline Mean & 16.7 & 16.5 & $8+8$ & $8+9$ & & & & \\
\hline
\end{tabular}

* cons, conservative; surg, surgery

Table II. Radiological findings in the 18 pairs*

\begin{tabular}{|c|c|c|c|c|c|c|c|c|c|c|c|c|}
\hline \multirow[b]{2}{*}{ Number of pair } & \multicolumn{2}{|c|}{$\operatorname{Mose}^{9}(\mathrm{~mm})$} & \multicolumn{2}{|c|}{$\mathrm{AHI}(\%)$} & \multicolumn{2}{|c|}{ SAR ( $\left.{ }^{\circ}\right)$} & \multicolumn{2}{|c|}{ ATD (mm) } & \multicolumn{2}{|c|}{ LLD (mm) } & \multicolumn{2}{|c|}{ Stulberg $^{11}$ classification } \\
\hline & Cons & Surg & Cons & Surg & Cons & Surg & Cons & Surg & Cons & Surg & Cons & Surg \\
\hline 1 & 10 & 8 & 64.4 & 75.8 & -15 & -18 & 2 & 10 & 3 & 14 & IV & IV \\
\hline 2 & 6 & 3 & 74.0 & 66.3 & 0 & 0 & 5 & 8 & 12 & 8 & III & III \\
\hline 3 & 6 & 0 & 67.1 & 82.6 & 0 & -5 & 13 & 5 & 15 & 2 & III & II \\
\hline 4 & 3 & 0 & 68.7 & 80.0 & 0 & -3 & 3 & -8 & 11 & 5 & III & II \\
\hline 5 & 2 & 0 & 82.1 & 84.7 & 0 & -10 & -2 & -2 & 23 & 5 & II & II \\
\hline 6 & 6 & 0 & 74.0 & 77.4 & 0 & 1 & 5 & 5 & 12 & 0 & III & II \\
\hline 7 & 4 & 2 & 75.6 & 78.9 & 0 & 0 & 1 & -2 & 18 & 14 & IV & II \\
\hline 8 & 3 & 2 & 72.0 & 82.4 & 0 & -12 & -7 & -9 & 11 & 9 & IV & III \\
\hline 9 & 10 & 2 & 65.0 & 82.8 & -14 & -3 & 6 & 11 & 22 & 4 & IV & III \\
\hline 10 & 10 & 8 & 64.4 & 86.8 & -15 & -16 & 2 & 5 & 3 & 10 & IV & III \\
\hline 11 & 10 & 0 & 61.2 & 87.4 & -20 & 0 & -2 & 5 & 20 & 13 & IV & II \\
\hline 12 & 2 & 1 & 78.2 & 92.4 & 0 & 0 & 0 & 3 & 21 & 15 & II & II \\
\hline 13 & 1 & 2 & 77.8 & 72.7 & 2 & -7 & 6 & 3 & 3 & 5 & II & II \\
\hline 14 & 4 & 1 & 72.5 & 88.2 & 0 & -8 & 3 & 3 & 13 & 3 & IV & II \\
\hline 15 & 2 & 1 & 76.5 & 82.9 & 2 & -5 & 5 & 8 & 15 & 3 & II & II \\
\hline 16 & 3 & 2 & 83.9 & 65.6 & 2 & -15 & 2 & -5 & 7 & 12 & III & II \\
\hline 17 & 8 & 1 & 68.4 & 80 & 0 & 0 & 6 & -2 & 5 & 8 & IV & II \\
\hline 18 & 4 & 0 & 70 & 79.4 & -5 & 3 & 2 & 5 & 12 & 5 & IV & II \\
\hline Mean & 5.2 & 1.8 & 72.0 & 80.4 & -3.5 & -5.4 & 2.8 & 2.4 & 12.6 & 7.5 & & \\
\hline Median & 4.0 & 1.0 & 72.3 & 81.2 & 0.0 & -4.0 & 2.5 & 4.0 & 12.0 & 6.5 & & \\
\hline
\end{tabular}

* AHI, acetabular head index; SAR, slope of acetabular roof; ATD, articulotrochanteric distance; LLD, leg-length discrepancy; cons conservative; surg, surgery

One patient was selected from each treatment group to create the pairs for our study. Each pair was matched for gender, sex, body mass index within \pm 2 , age at onset, the stage at the first visit (the initial, necrotic or regenerative), Catterall group (III, IV, head-at-risk), and severe subluxation and/or a metaphyseal cyst. Catterall group III was evaluated more strictly by measuring the epiphyseal height from a lateral radiographic view. Heights which were less than $75 \%$ of the epiphysis of the normal side were classified as group III. At follow-up each pair was assessed radiologically by Mose's method, ${ }^{9}$ by measurement of the acetabular- head index (AHI), the slope of the acetabular roof (SAR), ${ }^{10}$ the articulotrochanteric distance (ATD) and the leg-length discrepancy (LLD) and by the classification of Stulberg, Coopermann and Wallensten. ${ }^{11}$ The measurements in both the conservative and surgical groups were performed on radiographs taken between the ages of 16 and 20 years.

Statistical analysis. We used the Wilcoxon signed-rank test (Statistical Analysis System, Cary, North Carolina). The results were expressed as the mean with the $95 \%$ confidence interval (CI) and the median. A p value of $<0.05$ was considered to be significant. 


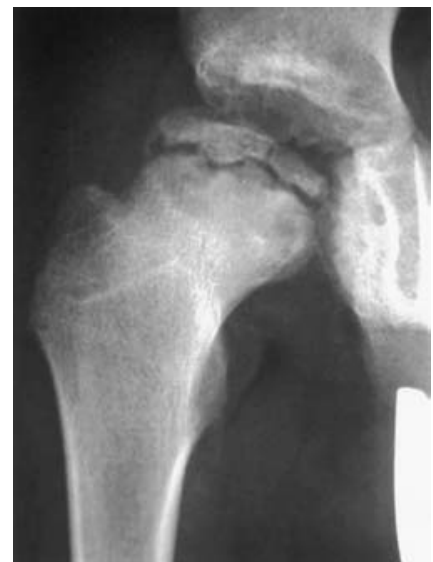

Fig. 1a

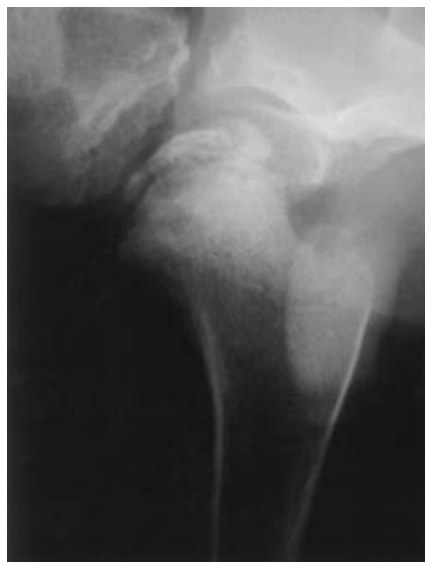

Fig. $1 b$

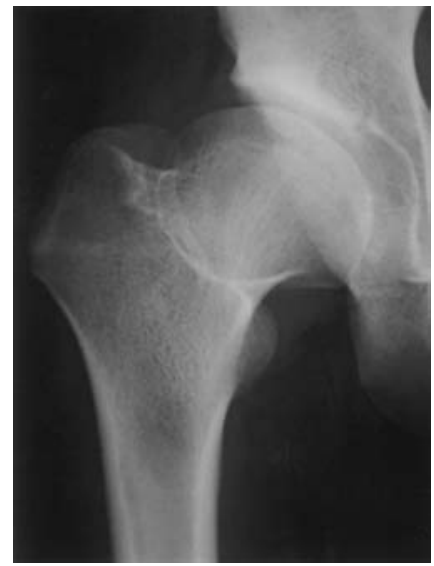

Fig. 1c

Plain radiographs of a seven-year-old boy (pair 4) in the conservative group with Catterall classification III and positive signs of subluxation and a metaphyseal cyst showing a) anteroposterior (AP) and b) lateral views and c) the same patient at the age of 19 years when classified as Stulberg class III.

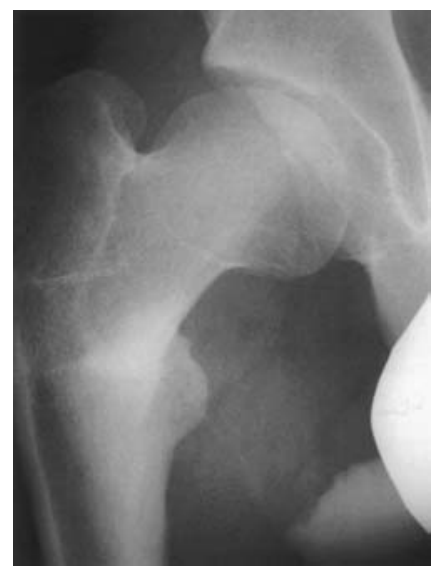

Fig. 2a

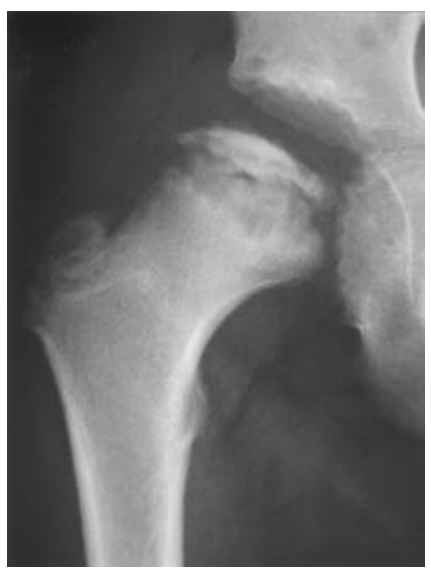

Fig. $2 b$

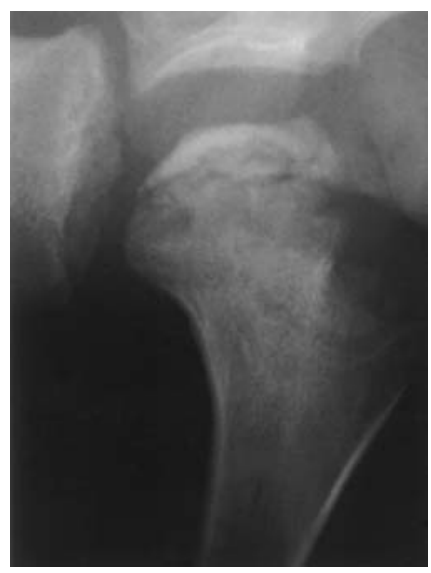

Fig. 2c

Radiographs of the paired surgical patient (pair 4) at 18 years of age showing a) a more spherical femoral head and classified as Stulberg class II, b) the AP and c) lateral views at onset.

\section{Results}

Eighteen pairs fitted the criteria. All were male. The most frequent stage at the first visit was the necrotic or fragmentation stage. Seventeen pairs were classified as Catterall group III and one as group IV. Subluxation and a metaphyseal cyst were seen in 14 pairs, and subluxation alone in four (Table I).

The results of the radiological measurements are given in Table II. The findings were significantly better for the surgical group compared with the conservative group for Mose's $\operatorname{method}(\mathrm{p}=0.0003)$, AHI $(\mathrm{p}=0.0096)$, and LLD $(\mathrm{p}=$ 0.0234).

No statistical difference was seen between the conservative and surgical groups in the SAR $(\mathrm{p}=0.245)$ and ATD $(\mathrm{p}=0.886)$. The mean confidence intervals $(\mathrm{CI})$ and median for the difference of each variable in each pair were $3.4 \mathrm{~mm}$ (2.0 to 4.8 ) and $2.5 \mathrm{~mm}$ for Mose's method, $-8.4 \%$ (-13.8\% to $-3.0 \%)$ and $-10.9 \%$ for the AHI, $1.9^{\circ}(-2.2$ to 6.0$)$ and $2.0^{\circ}$ for the SAR, $0.4 \mathrm{~mm}(-3.9$ to 4.7$)$ and $0.0 \mathrm{~mm}$ for the ATD, and $3.7 \mathrm{~mm}$ (1.0 to 6.4) and $6.0 \mathrm{~mm}$ for the LLD. Stulberg's classification showed that good results were more frequent in the surgical group than in the conservative group with better results in 12 pairs, the same result in six pairs and no worse results for any pair. Four hips in the conservative group were in Stulberg's class II, 5 in class III and 9 in class IV, while in the surgical group 13 were in class II, 4 in class III and 1 in class IV (Table II).

\section{Pair presentation}

Pair 4. The radiographs at presentation of a seven-year-old patient in the conservative group showed Catterall group 


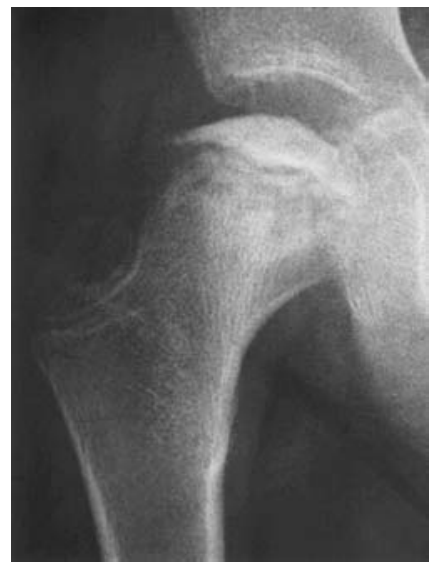

Fig. 3a

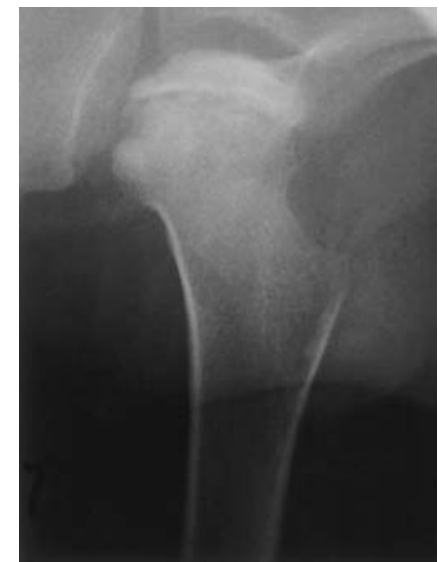

Fig. $3 b$
Radiographs of a patient aged six years and seven months (pair 9) in the surgical group in Catterall group IV with positive signs of subluxation and a metaphyseal cyst showing a) AP and b) lateral views.

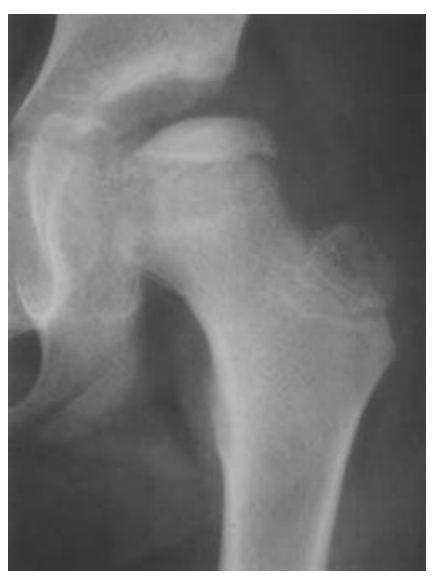

Fig. 4a

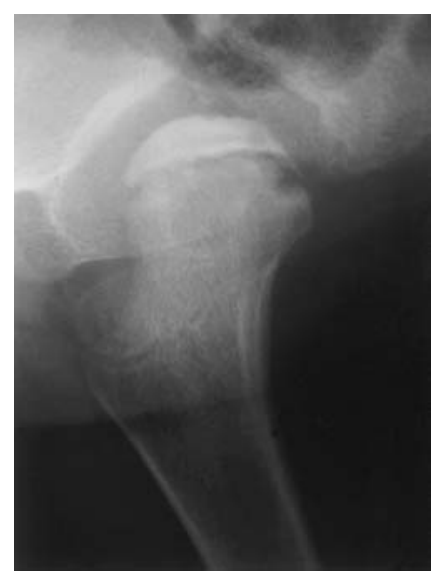

Fig. 4b

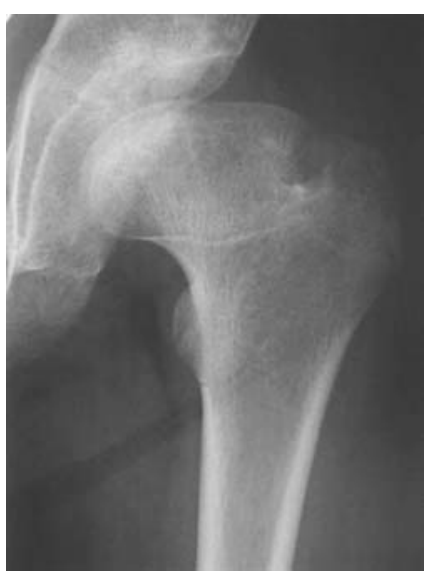

Fig. 4c

Radiographs of the paired conservative patient (pair 9), with the same classification and at-risk signs showing a) AP and b) lateral views and c) Stulberg class IV.

III with positive signs of subluxation and a metaphyseal cyst (Figs $1 \mathrm{a}$ and $1 \mathrm{~b}$ ). At 19 years of age the patient was classified as Stulberg class III with $3 \mathrm{~mm}$ by Mose's method and $68.7 \%$ of AHI (Fig. 1c). The radiograph of the paired surgical patient aged 18 years showed a more spherical femoral head ( $0 \mathrm{~mm}$ by Mose's method) and better acetabular cover $(80.0 \%$ of AHI) and was classified as Stulberg II (Fig. 2a). The appearance of this hip at onset is shown in Figures $2 \mathrm{~b}$ and $2 \mathrm{c}$. The LLD was $11 \mathrm{~mm}$ in the conservative patient and $5 \mathrm{~mm}$ in the surgical one. Neither patient had a positive Trendelenburg sign, in spite of the high greater trochanter. However, the SAR was slightly better in the conservative patient $\left(0^{\circ}\right)$ than in the surgical patient $\left(-3^{\circ}\right)$.

Pair 9. Both patients in this pair were classified as Catterall group IV. The surgical patient had initially been treated by a Tachdjian orthosis, followed by a varus femoral osteotomy because of radiological deterioration (Fig. 3). Radiographs for the conservative patient also had the same risk signs (Figs $4 \mathrm{a}$ and $4 \mathrm{~b}$ ). The results for this pair were quite different. The conservative patient was classified as Stulberg class IV (Fig. 4c) and the surgical patient as Stulberg class III (Fig. 5). The femoral head in the surgical patient was more spherical ( $2 \mathrm{~mm}$ by Mose's method) and well covered by the acetabulum $(82.8 \%$ of AHI), compared with 10 $\mathrm{mm}$ by Mose's method and $65 \%$ AHI in the conservative patient.

\section{Discussion}

The extent of the necrotic area and head-at-risk signs are generally accepted as two of the most influential factors affecting the prognosis in Perthes' disease. In our study, a modified form of Catterall's classification was used to assess the patient's prognosis. For this, the amount of viable bone and the height of the femoral head on the lateral view were measured in the necrotic or fragmentation stage and any head-at-risk signs were identified in the femoral head. 


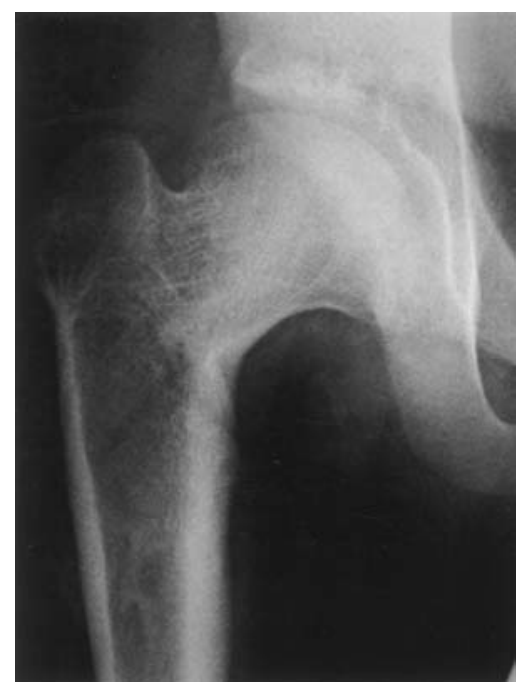

Fig. 5

A radiograph of the surgical patient classified as Stulberg class III (pair 9).

Herring et $\mathrm{al}^{12}$ reported the lateral pillar classification in which the height of the lateral part of the epiphysis (lateral pillar) is estimated during the fragmentation stage. It has been widely accepted because of its simplicity and reproducibility. However, Lappin, Kealey and Cosgrove ${ }^{13}$ have suggested that the Herring grade often changes with time, especially in the early stages of the disease. We therefore decided not to use this method of classification.

The indications for surgical treatment in Perthes' disease remain controversial despite several reports comparing the results of conservative and surgical treatment. ${ }^{1-6}$

In our study by using matched pairs of conservatively and surgically treated patients we have tried to obtain a more rigorously controlled comparison between the two groups. We included only patients classified at presentation as Catterall group III and IV.

Lloyd-Roberts et $\mathrm{al}^{4}$ stated that containment by femoral osteotomy was the treatment of choice in patients with atrisk signs provided that severe deformity had not already occurred. However, their study of three groups, an osteotomy, an untreated, and an uncontained treated group was based on a simplistic standard of patient selection and only excluded Catterall group-I patients. Marklund and Tilberg ${ }^{5}$ compared conservative treatment in one institute and surgical treatment in another with differing guidelines of treatment. Lahdes-Vasama et $\mathrm{al}^{3}$ compared the outcome between conservative and surgical groups in terms of the femoral sphericity, acetabular cover and acetabular direction. However, they selected patients for both groups by using only the classification A or B described by Salter and Thompson. ${ }^{14}$ Randomised or simple mass studies do not allow us to make an accurate judgement of the correct choice of treatment. The use of carefully matched pairs of conservatively and surgically-treated patients in our study may help to resolve this important issue which has long been discussed without any general consensus.

Some authors have reported that the most important factor for the long-term prognosis is the sphericity of the femoral head., 11,15 In our study sphericity of the head measured by Mose's method and the AHI, which reflects acetabular congruity were significantly better in the surgical group. Lecuire ${ }^{16}$ has stated in a follow-up study of more than 50 years that normal or flattened spherical heads present few problems, but irregular or very irregular heads are associated with a poor result. There was no statistical difference in SAR or ATD between the groups.

Weiner, Weiner and Riley ${ }^{17}$ recommended a post-operative femoral varus angle of more than $105^{\circ}$. Heikkinen and Puranen ${ }^{18}$ suggested that 100 to $110^{\circ}$ of varus were optimal for obtaining good containment of the femoral head in the acetabulum. We usually performed osteotomies with about $40^{\circ}$ of varus which resulted in a neck-shaft angle of approximately $100^{\circ}$. It is generally thought that a femoral varus osteotomy produces a higher position of the greater trochanter, with a greater LLD than conservative treatment. However, our results do not support this belief, in terms of the ATD and LLD. The value of the ATD in both treatment groups did not show a statistical difference $(\mathrm{p}=0.886)$. The value of the LLD showed a statistically better result for the surgical group $(\mathrm{p}=0.0234)$ contrary to our expectations. This suggests that even in the conservative group, a considerable loss of epiphyseal height had occurred, resulting in poor values for the ATD and LLD. Yrjönen ${ }^{15}$ reported that the incidence of LLD was similar in conservative and surgical groups. The results of our study suggest that femoral osteotomy does not cause increased LLD in patients with Catterall group-III disease compared with conservative treatment. Clinically the higher position did not give rise to a Trendelenburg phenomenon in either group.

We conclude that surgical treatment can improve the sphericity of the femoral head and provide greater acetabular cover than conservative treatment. However, the SAR was not affected. A greater degree of LLD and a smaller ATD were not seen in the surgical groups. Our study suggests that surgical instead of conservative treatment is preferable for patients with severe Perthes' disease.

Although only 18 strictly matched pairs (36 hips) were included in our study, the results suggest that varus femoral osteotomy is better than conservative treatment in patients with severe (Catterall group III or group IV) Perthes' disease.

No benefits in any form have been received or will be received from a commercial party related directly or indirectly to the subject of this article.

\section{References}

1. Evans IK, Deluca PA, Gage JR. A comparative study of ambulation abduction bracing and varus derotation osteotomy in the treatment of severe Legg-Calvé-Perthes disease in children over 6 years of age. J Pediatr Orthop 1988;8:676-82.

2. Fulford GE, Lunn PG, Macnicol MF. A prospective study of nonoperative and operative management for Perthes' disease. J Pediatr Orthop 1993;13:281-5.

3. Lahdes-Vasama TT, Marttinen EJ, Merikanto JEO. Outcome of Perthes' disease in unselected patients after femoral varus osteotomy and splintage. J Pediatr Orthop B 1997;6:229-34. 
4. Lloyd-Roberts GC, Catterall A, Salamon PB. A controlled study of the indications for and the results of femoral osteotomy in Perthes's disease. J Bone Joint Surg [Br] 1976;58-B:31-6.

5. Marklund T, Tillberg B. Coxa plana: a radiological comparison of the rate of healing with conservative measures and after osteotomy. J Bone Joint Surg [Br]1976;58-B:25-30.

6. Vukasinovic Z, Slavkovic S, Milickovic S, Siqeca A. Combined Salter innominate osteotomy with femoral shortening versus other methods of treatment for LeggCalvé-Perthes disease. J Pediatr Orthop B 2000;9:28-33.

7. Kamegaya M. Comparative study of Perthes's disease treated by various ambulatory orthoses. Nippon Seikeigeka Gakkai Zasshi 1987;61:917-32.

8. Catterall A. The natural history of Perthes' disease. J Bone Joint Surg [Br] 1971;53-B 37-53.

9. Mose K, Hjorth L, Ulfeldt M, Christensen ER, Jensen A. Legg-Calvé-Perthes' disease: the late occurrence of coxarthrosis. Acta Orthop Scand Supp/1977;169:1-39.

10. Stulberg SD, Harris WH. Acetabular dysplasia and development of osteoarthritis of hip [abstract]. In: Procs second open scientific meeting of the Hip Society. Mosby: St Louis 1974:82-93
11. Stulberg SD, Coopermann DR, Wallensten R. The natural history of Legg-CalvéPerthes disease. J Bone Joint Surg [Am] 1981;63-A:1095-108.

12. Herring JA, Neustaft JB, Williams JJ, Early JS, Browne RH. The lateral pillar classification of Legg-Calvé-Perthes disease. J Pediatr Orthop 1992;12:143-50.

13. Lappin K, Kealey D, Cosgrove A. Herring classification: how useful is the initia radiograph? J Pediatr Orthop 2002;22:479-82

14. Salter RB, Thompson GH. Legg-Calvé-Perthes: the prognostic significance of the subchondral fracture and a two-group classification of the femoral head involvement. J Bone Joint Surg [Am] 1984;66-A:479-89.

15. Yriönen T. Long-term prognosis of Legg-Calvé-Perthes disease: a meta-analysis J Pediatr Orthop B 1999;8:169-72.

16. Lecuire $\mathbf{F}$. The long-term outcome of primary osteochondritis of the hip (Legg-CalvéPerthes' disease). J Bone Joint Surg [Br] 2002;84-B:636-40.

17. Weiner SD, Weiner DS, Riley PM. Pitfalls in treatment of Legg-Calvé-Perthes disease using proximal femoral varus osteotomy. J Pediatr Orthop 1991;11:20-4.

18. Heikkinen E, Puranen J. Evaluation of femoral osteotomy in the treatment of LeggCalvé-Perthes' disease. Clin Orthop 1980;150:60-8 